\title{
Should the host reaction to anisakiasis influence the treatment? Different clinical presentations in two cases
}

\author{
Stefano Pontone, Giovanni Leonetti, Eleonora Guaitoli, Renzo Mocini, Simone Manfredelli, \\ Antonio Catania, Paolo Pontone and Salvatore Sorrenti
}

Department of Surgical Sciences. “Sapienza” University of Rome. Italy

\begin{abstract}
Gastrointestinal anisakiasis is a parasitic infection occurring in people that consume raw or inadequately cooked fish or squid. It is frequently characterized by severe epigastric pain, nausea and vomiting caused by the penetration of the larvae into the gastric wall. Acute gastric anisakiasis with severe chest discomfort is rarely reported in Italy. On the other hand, gastro-allergic anisakiasis with rash, urticaria and isolated angioedema or anaphylaxis is a clinical entity that has been described only recently. Also, if patients usually develop symptoms within 12 hours after raw seafood ingestion, not always endoscopic exploration can promptly identify the Anisakis larvae. Moreover, some authors consider the prevailing allergic reaction as a natural and effective defense against the parasitic attack. We report two cases of peculiar manifestations of anisakiasis in both acute and chronic forms (severe chest discomfort and anaphylactoid reaction).
\end{abstract}

Key words: Anisakis simplex. Chest pain. Helminthic infections. Gastric perforation. Anaphylaxis. Raw fish.

\section{INTRODUCTION}

Gastrointestinal Anisakiasis (GA) is a parasitic disease acquired by infection with Anisakis simplex (As) or Pseudoterranova decipiens nematodes belonging to the Anisakidae family (1). This parasitic disease occurs most

Pontone S, Leonetti G, Guaitoli E, Mocini R, Manfredelli S, Catania $A$, Pontone $P$, Sorrenti S. Should the host reaction to anisakiasis influence the treatment? Different clinical presentations in two cases. Rev Esp Enferm Dig 2012;104:607-610.

Received: 27-09-2012

Accepted: $18-10-2012$

Correspondence: Stefano Pontone. Department of Surgical Sciences. "Sapienza" University of Rome. Italy

email: stefano.pontone@uniroma1.it commonly where people consume raw or inadequately cooked fish or squid (2). In particular, most cases have been described in Japan and in the coastal regions of Europe, especially the Netherlands, Germany, France, Spain (3), Italy and the United States. Actually, 12 cases of gastric anisakiasis have been described in Italy (4). The risk of anisakiasis has increased proportionally with the increase in the consumption of sushi, sashimi, raw and smoked fish dishes. In Italy, the homemade marinated anchovies are the most frequent cause of clinical presentation (5). In infected individuals four major clinical syndromes are described, including gastric, intestinal, ectopic and allergic diseases (6). The most common presentation, the GA, is frequently characterized by severe epigastric pain, nausea, vomiting and caused by penetration of the larvae into the gastrointestinal wall. Thus, the patient is admitted to the surgical area of the emergency department, even if a multidisciplinary approach would be required. Usually, patients develop symptoms within 12 hours after the ingestion of raw seafood. In cases where upper endoscopy is performed within few hours or days after ingestion, endoscopic findings show gastric reddening, edema and erosions or ulcerations around the worm penetration site, which may frequently affect the gastric greater curvature. Endoscopic examination, when delayed, can highlight a chronic mucosal change with a greater difficulty in anisakiasis detection. An allergic response can be associated with rash (7), urticaria, isolated angioedema or anaphylaxis $(8,9)$. The endoscopic worm extraction is usually followed by a rapid improvement in symptoms. However, a delayed treatment may allow the larvae to penetrate into the submucosa. While intestinal or extraintestinal infections can required surgical removal of the larvae, the diagnosis of GA obtained with a non-invasive method allows a conservative treatment by supportive therapy or albendazole administration (10-12). Chest discomfort during anisakiasis was rarely reported $(13,14)$. We report two cases of severe anisakiasis presentation due to acute and chronic infections. 


\section{CASE REPORT 1}

A 55-year old woman with acute chest discomfort was admitted to our hospital. Her medical history showed no evidence of specific diseases. The patient suddenly complained of the onset of a vague sensation of chest constriction that subsequently became severe and persisting. She denied abdominal pain. On admission, physical examination showed distended abdomen with blood pressure, pulse, heart and respiratory parameters within the normal limits. Serial blood tests showed a blood cell count of 11,000 leucocytes and $2 \%$ of eosoniphils. Electrocardiogram, echocardiogram, chest X-ray and abdominal ultrasound were all normal. A coronary contrast TC, performed three hours later, showed no abnormalities but, as an incidental finding, a localized thickening of the gastric fundus wall was demonstrated. A careful endoscopic examination revealed a superficial ulcer in the fundus wall gastric, with a parasitic worm penetrating the gastric mucosa (Fig. 1). Thus, the worm's invasion of the gastric fundus can explain acute chest discomfort instead of gastric symptoms. A single worm was removed with biopsy forceps and multiple biopsies were performed close to the penetration site. Histological examination of the specimen showed chronic inflammation with a marked increase in eosinophils and a foreign body was assumed to be a part of the As larvae. We insisted on asking the patient about the ingestion of undercooked fish and she confirmed that 12 hours before she had eaten a portion of marinated anchovies. An anti-anisakis larvae immunoglobulin $\mathrm{G}$ antibody test was performed using an Elisa test and the result was positive. The patient condition immediately improved after removing the worm. She was discharged after receiving supportive care. The 12-month follow-up was negative.

\section{CASE REPORT 2}

A 28-year old man was admitted to our Emergency Department to study an epigastric pain associated with diarrhea. The medical history was negative for both medication and food allergy. Blood tests were unaltered $(\mathrm{Hb} 15.8 \mathrm{~g} / \mathrm{dl}$, WBC 8000, N 64.3\%) as well as the abdominal X-ray and ultrasounds. The patient reported the onset of a flank rash a month earlier (Fig. 2) in association with angioedema (Fig. 3) and urticaria, which was successfully treated with chlorphenamine maleate. Because of the reported episode, the patient underwent immunological screening, showing positive anti-anisakis larvae immunoglobulin $\mathrm{G}$ antibody (IgE $140 \mathrm{kU} / \mathrm{l}$, As specific IgE 24,40 kUA/l). The endoscopic examination performed within 12 hours after admission revealed no presence of the As larvae but highlighted some slight ulcerations in the large gastric curvature suggesting previous parasitism (Fig. 4). Multiple biopsies were performed at the level of the detected lesions without leading to any specific diagnosis but to a nonspecific hyperemia with eosinophilic infiltration. Considering the clinical

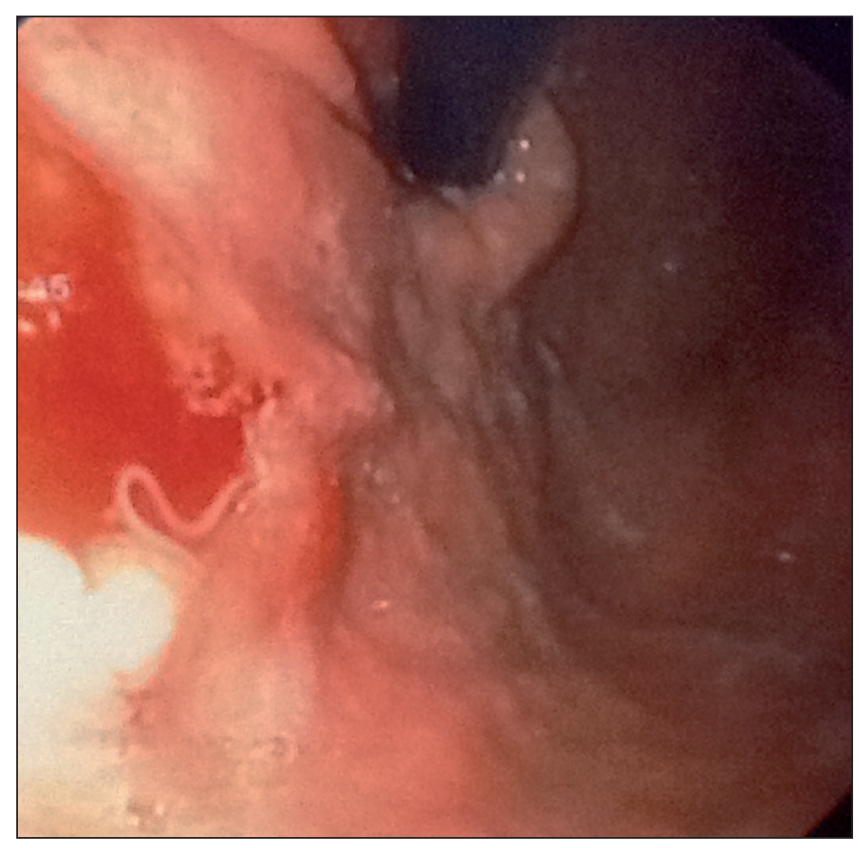

Fig. 1. Anisakis larvae close to erosion of the gastric fundus with active bleeding.

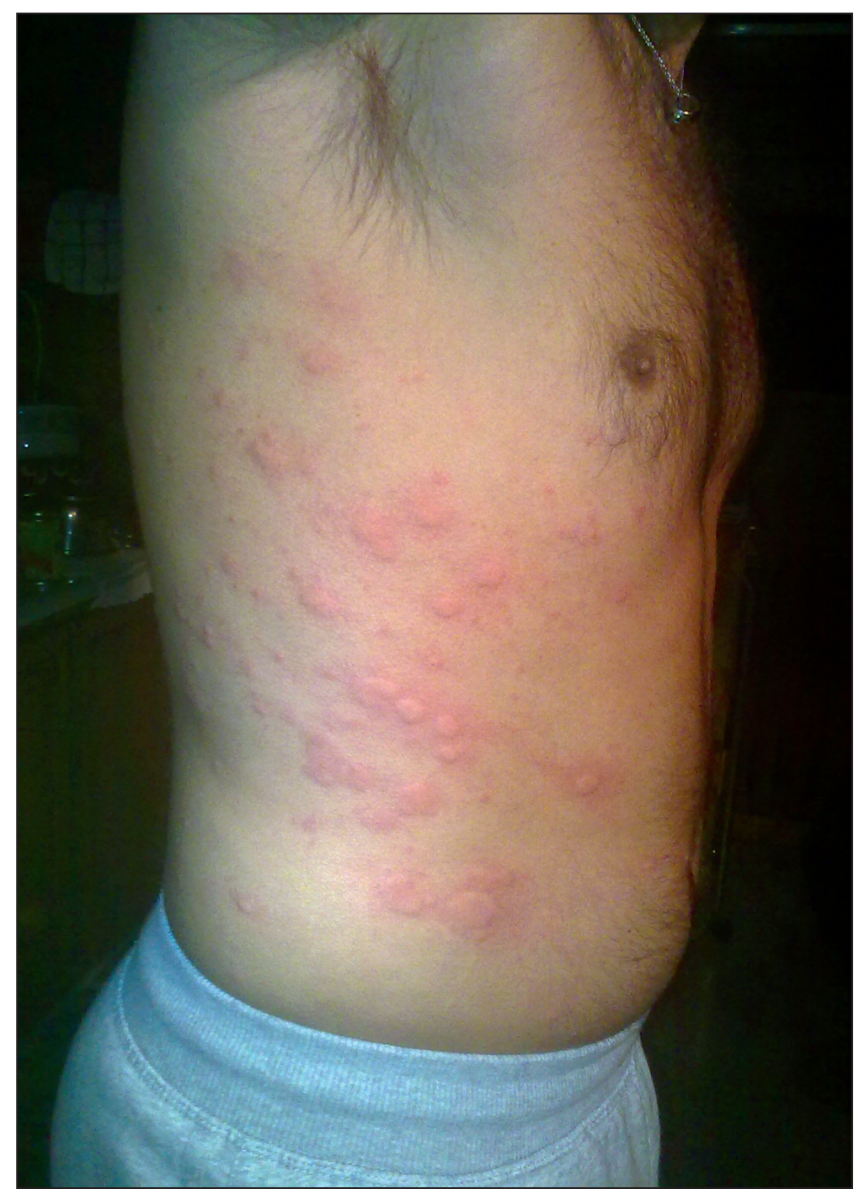

Fig. 2. Diffuse flank rash linked to urticarial reaction during the first contact with the Anisakis larvae in case report 2. 


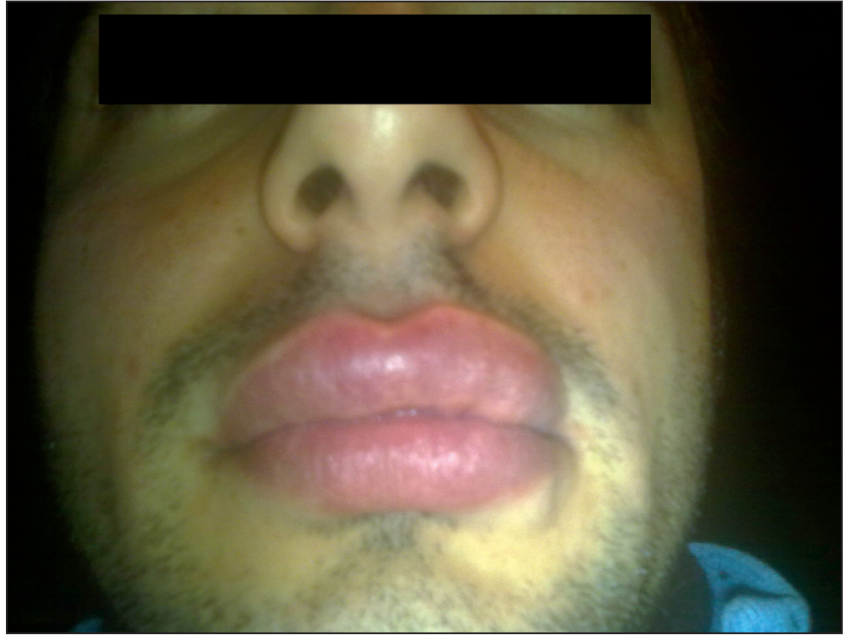

Fig. 3. Angioedema at the first contact with the Anisakis larvae in case report 2.

improvement, the patient was discharged with a home therapy (Albendazole $100 \mathrm{mg} /$ day for 7 days) three days after admission. After a follow-up of 6 months, the patient reported no similar episodes and anisakiasis serology was negative.

\section{DISCUSSION}

Larval anisakiasis was firstly reported in 1960 by Van Thiel et al. (14). Humans become infected by ingestion of the As larvae, which are present in seafood dishes. Many cases have been reported in Japan (1) where there is a high rate of consumption of raw fish but the disease has been recognized in some other countries (15). Herring, cod, squid, mackerel, tuna and cuttlefish are the most commonly potential larval conductors. Major clinical syndromes in infected people include gastric, intestinal, ectopic and allergic disease, with gastric anisakiasis being the most common. GA is characterized by severe epigastic pain, nausea, vomiting and caused by penetration of the larvae into the gastrointestinal wall (7). Instead, gastro-allergic anisakiasis (GAA) is represented by an allergic reaction provoked by the As penetration through the gastric mucosa recently described $(16,17)$. Thus, in our two cases, despite the rarity of parasitism in our country, both manifestations occurred, each with some peculiarities (severe chest discomfort and anaphylactoid reaction). Considering the fundamental importance of the accurate medical history, we would like to stress that specific serological positivity and the presence of gastric lesions are found in both forms of the condition. On the contrary, according to the different symptoms and endoscopic examination, we can speculate that we have encountered the same disease in two phases. Thus, as described in the second case, our intervention occurred remotely from the primary infection that manifested itself by the allergic reaction and apparently without any gastric symptoms. This entity, characterized by urticaria and angioedema without sur-

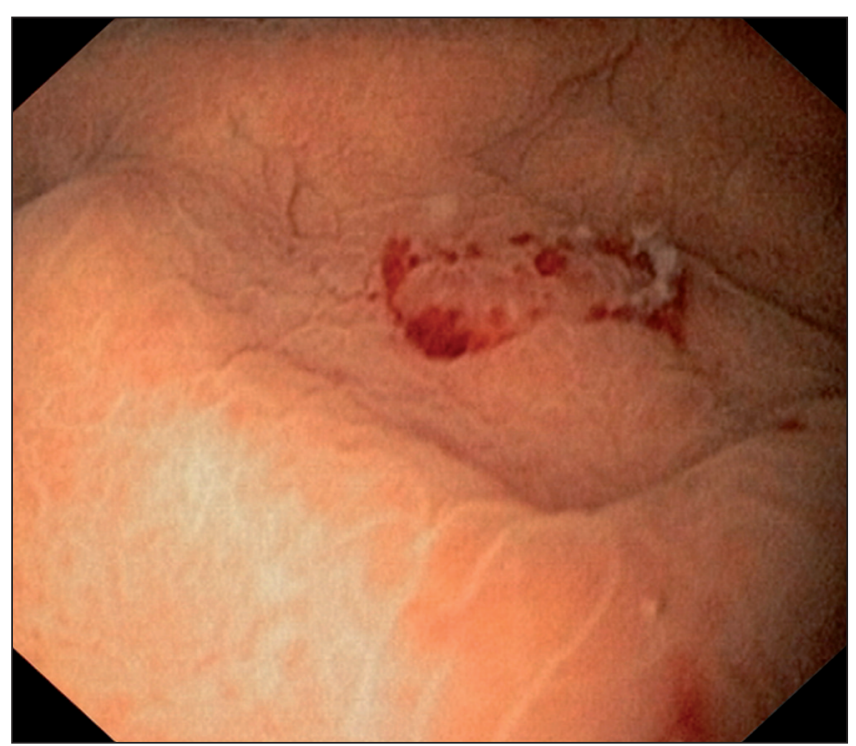

Fig. 4. The "water immersion" technique highlighting a slight ulceration in the large gastric curvature in case report 2.

gical complications, occurs when the larva penetrates only superficially into the gastric mucosa. In this case, the chance of identifying the parasite in the gastric cavity during endoscopic examination is very poor. In the first case, instead, the chest discomfort caused by GA was so severe that the patient had to go to the Emergency Department. Occurring within 6 hours after parasite ingestion and contact, endoscopic examination was effective as a diagnostic confirmation and curative treatment, considering that, in our case, the endoscopic removal of the worm was followed by rapid improvement in symptoms. Our cases support Daschner's (17) thinking according to which the allergic reaction is a price to pay for the rapid expulsion of the parasite. On this basis, we could explain that, in the first case, the patient had surgical complications without any allergic reactions and, in the second case, the patient was admitted to our unit at the time of the second contact with the parasite. The allergic reaction may have hindered the permanence of the larva in the stomach and its penetration through the gastric wall, thus making diagnosis impossible. The clinical implication of these considerations is reflected on the patient management. In fact, the GAA clinical manifestations rather than the gastric or "surgical" symptoms may authorize, if this hypothesis is confirmed, to adopt a "wait and see" treatment.

\section{REFERENCES}

1. Ishikura H. General survey of anisakis and anisiakis. In: Ishihara $H$, Namiki M, eds. Gastric Anisakiasis in Japan. Epidemiology, Diagnosis, Treatment. Tokyo: Springer-Verlag; 1988. p. 3-11.

2. Cabrera R. Anisakiasis outbreak by Anisakis simplex larvae associated to Peruvian food in Spain. Rev Esp Enferm Dig 2010;102:610-1.

3. Fernández Salazar LI, Guantes de Vigo B, Herreros Rodríguez J, Abril Vega C, Calabia del Campo J, de la Calle Valverde F, et al. Another multiple gastric anisakiasis case. Rev Esp Enferm Dig 2010;102:60-1. 
4. Zullo A, Hassan C, Scaccianoce G, Lorenzetti R, Campo SM, Morini S. Gastric anisakiasis: Do not forget the clinical history! J Gastrointestin Liver Dis 2010;19:359.

5. AAITO-IFIACI Anisakis Consortium. Anisakis hypersensitivity in Italy: prevalence and clinical features: A multicenter study. Allergy 2011;66:1563-9.

6. Hochberg NS, Hamer DH. Anisakidosis: Perils of the deep. Clin Infect Dis 2010;51:806-12.

7. Kakizoe S, Kakizoe H, Kakizoe K, Kakizoe Y, Maruta M, Kakizoe T. Endoscopic findings and clinical manifestation of gastric anisakiasis. Am J Gastroenterol 1995; 90:761-3.

8. López-Serrano MC, Gomez AA, Daschner A, Moreno-Ancillo A, de Parga JM, Caballero MT, et al. Gastroallergic anisakiasis: Findings in 22 patients. J Gastroenterol Hepatol 2000;15:503-6.

9. Foti C, Nettis E, Cassano N, Di Mundo I, Vena GA. Acute allergic reactions to Anisakis simplex after ingestion of anchovies. Acta Derm Venereol 2002;82:121-3.

10. Nawa Y, Hatz C, Blum J. Sushi delights and parasites: the risk of fishborne and foodborne parasitic zoonoses in Asia. Clin Infect Dis 2005;41:1297-303.
11. Pacios E, Arias-Diaz J, Zuloaga J, Gonzalez-Armengol J, Villarroel P, Balibrea JL. Albendazole for the treatment of anisakiasis ileus. Clin Infect Dis 2005;41:1825-6.

12. Moore DA, Girdwood RW, Chiodini PL. Treatment of anisakiasis with albendazole. Lancet 2002;360:54.

13. Sugano S, Suzuki T, Kagesawa M, Ohshima Y. Noncardiac chest pain due to acute gastric anisakis. Dig Dis Sci 1993;38:1354-6.

14. Van Thiele PH, Kuipers FC, Roskamm RTH. A nematode parasitic, to herring, causing acute abdominal syndromes in man. Trop Geogr Med 1960;12:97-113

15. Kliks MM. Anisakiasis in the western United States: four new case reports from California. Am J Trop Med HYG 1983;32:526-32.

16. Daschner A, Cuéllar $\mathrm{C}$. The hidden sense of symptoms: Urticaria can be beneficial. Med Hypotheses 2010;75:623-6

17. Daschner A, Alonso-Gómez A, Cabañas R, Suarez-de-Parga JM, López-Serrano MC. Gastroallergic anisakiasis: Borderline between food allergy and parasitic disease-clinical and allergologic evaluation of 20 patients with confirmed acute parasitism by Anisakis simplex. J Allergy Clin Immunol 2000;105:176-81. 